\title{
Representation of the Hubbard model in the Clifford algebra
}

\author{
Francisco Guil ${ }^{1}$, Gabriel Alvarez \\ Departamento de Física Teórica, Facultad de Ciencias Físicas, Universidad Complutense, 28040 Madrid, \\ Spain
}

Received 2 October 1995; accepted 1 November 1995

\begin{abstract}
A new representation of the Hubbard model in the Clifford algebra specially suited to describe complex many-particle states in simple terms is introduced. The form of the hamiltonian and other relevant observables in the new representation are discussed and several families of nontrivial halffilled states are readily obtained.
\end{abstract}

PACS: 75.10.Lp, 02.20.Qs

Keywords: Hubbard, Representation Theory

\section{Introduction: The Hubbard model}

The aim of this paper is to introduce a new representation of a well-known system, the Hubbard model of itinerant electrons moving on a graph [1,2]. This representation is specially suited to obtain compact expressions for many-particle states, among which we find some interesting half-filled states. Our results are valid in any dimension, do not rely on the bipartite character of the graph and are applicable to a somewhat broader class of generalized Hubbard models specified below.

Following the standard conventions $[1,2]$, we consider a graph $L$ with $\ell$ sites and hamiltonian $H=T+V$. The kinetic energy operator $T$ is given in second-quantized form by

\footnotetext{
${ }^{1}$ Corresponding author. Fax: 34-1-3945197.

E-mail: guil@lisa.fis.ucm.es 


$$
T=\sum_{\sigma=\uparrow, \downarrow} \sum_{i, j=1}^{\ell} t_{i j} c_{i \sigma}^{+} c_{j \sigma}
$$

with $t_{i j}=t_{j i}^{*}$ and $t_{i i}=0$ (the nonvanishing hopping matrix elements $t_{i j} \neq 0$ define the edges or bonds connecting pairs of vertices in $L), \sigma=\uparrow, \downarrow$ denote the spin states, $c_{i \sigma}^{+}$is the creation operator for an electron at site $i$ with spin $\sigma$, and the creation and destruction operators satisfy the canonical anticommutation relations

$$
\begin{aligned}
& c_{i \sigma}^{+} c_{j \tau}+c_{j \tau} c_{i \sigma}^{+}=\delta_{i j} \delta_{\sigma \tau}, \\
& c_{i \sigma}^{+} c_{j \tau}^{+}+c_{j \tau}^{+} c_{i \sigma}^{+}=0, \\
& c_{i \sigma} c_{j \tau}+c_{j \tau} c_{i \sigma}=0 .
\end{aligned}
$$

The operator $V$ represents the potential energy, which can be written in terms of the mutually commuting number operators

$$
\begin{aligned}
n_{i} & =n_{i \uparrow}+n_{i \downarrow}, \\
n_{i \sigma} & =c_{i \sigma}^{+} c_{i \sigma} .
\end{aligned}
$$

For the standard short-range Hubbard model the potential energy is

$$
V=\sum_{i=1}^{\ell} V_{i}\left(n_{i \uparrow}-\frac{1}{2}\right)\left(n_{i \downarrow}-\frac{1}{2}\right)=\frac{1}{2} \sum_{i=1}^{\ell} V_{i}\left(n_{i}-1\right)^{2}-\frac{1}{4} \sum_{i=1}^{\ell} V_{i}
$$

although our results are valid for any polynomial potential $V\left(n_{1}, \ldots, n_{\ell}\right)$ in the $n_{i}$. Different choices of this polynomial define various generalized Hubbard models $[1,2]$. The space of states where the creation and destruction operators act - the Fock space is

$$
\mathcal{H}=\mathbf{C}\left\{s_{\alpha \beta}=c_{\uparrow}^{+\alpha} c_{\downarrow}^{+\beta}|0\rangle\right\}
$$

where $\alpha=\left(\alpha_{1}, \ldots, \alpha_{\ell}\right), \beta=\left(\beta_{1}, \ldots, \beta_{\ell}\right), \alpha_{i}, \beta_{i} \in\{0,1\}$ are multi-indices, $c_{\uparrow}^{+\alpha}=$ $c_{1 \uparrow}^{+\alpha_{1}} c_{2 \uparrow}^{+\alpha_{2}} \ldots c_{\ell \uparrow}^{+\alpha_{\ell}}$ as usual, and we assume that the states $s_{\alpha \beta}$ are an orthonormal set determining the Hilbert space structure in $\mathcal{H}\left(\operatorname{dim} \mathcal{H}=4^{\ell}\right)$. The time-independent Schrödinger equation for our hamiltonian reads

$$
H \psi=E \psi,
$$

and its solutions admit as quantum numbers the total number of particles, the (square of the) total spin $s(s+1)$, and its $z$-component $s_{z}$, which are

$$
\begin{aligned}
& N=\sum_{i=1}^{\ell} n_{i}, \\
& S_{z}=\frac{1}{2} \sum_{i=1}^{\ell}\left(n_{i \uparrow}-n_{i \downarrow}\right),
\end{aligned}
$$




$$
S^{2}=S_{z}^{2}+\frac{1}{2}\left(S_{+} S_{-}+S_{-} S_{+}\right),
$$

where $S_{+}=\sum_{i=1}^{\ell} c_{i \uparrow}^{+} c_{i \downarrow}$ and $S_{-}=S_{+}^{+}$. These operators are generators of the spin algebra

$$
g l(2) \cong \mathbf{C}\left\{N, S_{2}, S_{+}, S_{-}\right\}
$$

with center $N$, which is a symmetry algebra for the hamiltonian $H$.

Our first task in the next section will be to identify the Fock space with a geometric Hilbert space, namely the exterior algebra over the vector space $E$ of single-particle states. In this context, the compatibility of the system of observables $\left\{H, N, S_{z}, S^{2}\right\}$ turns out to be a consequence of the behavior of the exterior algebra with respect to the operation of direct sum of subspaces, and we are able to describe explicitly the reduction of $H$ in terms of the Lie algebra $g l(\ell)$. This analysis of the Hubbard model in the frame of standard representation theory [3] provides the basics for the main result of the paper, a new representation in which half the variables are eliminated and the states become operators in a Clifford algebra. This is accomplished in Section 3, where the corresponding expressions for the main observables (including the hamiltonian) are derived with an invariant (i.e., basis-independent) argument. Furthermore, this representation is naturally associated with a decomposition into invariant subspaces under the action of the hamiltonian (roughly, the concepts of spin-up and spin-down are replaced by pairs and holes) whose relation to the usual formulation is discussed. Finally, in Section 4 , we show the advantages of treating more symmetrically observables and states by deriving a few direct consequences: the structure of the Schrödinger equation in this representation immediately suggests several families of nontrivial half-filled states which are superpositions of states with one hole and one pair. Some of these states seem to play a major role as building blocks for more general half-filled states.

\section{Representation theory}

The starting point of our discussion is the fact that the abstract Fock space $\mathcal{H}$ can be conveniently identified with the exterior algebra $\Lambda(E)=\bigoplus_{k=0}^{2 \ell} \Lambda^{k}(E)$ of the space $E$ of single-particle states ( $\operatorname{dim} E=2 \ell$ ) with scalar product given by

$$
\left(u_{1} \wedge \ldots \wedge u_{k_{1}}, v_{1} \wedge \ldots \wedge v_{k_{2}}\right)=\delta_{k_{1} k_{2}} \operatorname{det}\left(u_{i}, v_{j}\right),
$$

where $\left(u_{i}, v_{j}\right)$ is the scalar product in $E$ [4]. For each vector $u \in E$ there exists an exterior-multiplication operator (also denoted by $u$ ) defined by

$$
u: \xi \in \Lambda(E) \rightarrow u \xi=u \wedge \xi \in \Lambda(E)
$$

and an inner-derivation operator denoted $\partial / \partial u$ and defined by

$$
\begin{gathered}
\frac{\partial \xi}{\partial u}=(u, \xi), \quad \xi \in \Lambda^{1}(E), \\
\frac{\partial}{\partial u}(\xi \wedge \eta)=\frac{\partial \xi}{\partial u} \wedge \eta+(-1)^{k} \xi \wedge \frac{\partial \eta}{\partial u}, \quad \xi \in \Lambda^{k}(E),
\end{gathered}
$$


and zero on $\Lambda^{0}(E)$. It is easy to check that $\partial / \partial u=u^{+}$with respect to the scalar product (2.1). Let us consider now the decomposition of the single-particle space $E$ into spinup and spin-down subspaces, $E=E_{\uparrow} \oplus E_{\downarrow}, E_{\uparrow} \perp E_{\downarrow}$, $\operatorname{dim} E_{\uparrow}=\operatorname{dim} E_{\downarrow}=\ell$, and pick orthonormal bases

$$
\begin{aligned}
& \left\{x_{1}, \ldots, x_{\ell}\right\} \subset E_{\uparrow}, \\
& \left\{y_{1}, \ldots, y_{\ell}\right\} \subset E_{\downarrow},
\end{aligned}
$$

which provide explicit isomorphisms $E_{\uparrow} \cong E_{\downarrow} \cong \mathbf{C}^{\ell}$. The above-mentioned identification is furnished by the correspondence where $|0\rangle \leftrightarrow 1 \in \Lambda^{0}(E) \cong \mathbf{C}, s_{\alpha \beta} \leftrightarrow x^{\alpha} y^{\beta}$, and the creation operators $c_{i \uparrow}^{+}$and $c_{i \downarrow}^{+}$are represented by the exterior-multiplication operators $x_{i}$ and $y_{i}$ respectively. The first canonical anticommutation relation (1.1) reads

$$
x_{i} \frac{\partial}{\partial x_{j}}+\frac{\partial}{\partial x_{j}} x_{i}=\delta_{i j}
$$

the number operators are

$$
n_{i}=x_{i} \frac{\partial}{\partial x_{i}}+y_{i} \frac{\partial}{\partial y_{i}}
$$

and the hamiltonian is

$$
H=\sum_{i, j=1}^{\ell} t_{i j}\left(x_{i} \frac{\partial}{\partial x_{j}}+y_{i} \frac{\partial}{\partial y_{j}}\right)+V\left(n_{1}, \ldots n_{\ell}\right) .
$$

Due to the symmetric way in which the Hubbard model treats spin-up and spin-down, the hamiltonian belongs to the enveloping algebra of the Lie algebra $g l(\ell)$, and the space $\Lambda(E)=\Lambda\left(E_{\uparrow} \oplus E_{\downarrow}\right)$ is a module of $g l(\ell)$ : to each $\ell \times \ell$ matrix $a$ one can assign the operator

$$
\begin{aligned}
u_{1} \wedge \ldots \wedge u_{n} \mapsto & \left(\xi_{a} u_{1}\right) \wedge \ldots \wedge u_{n}+u_{1} \wedge\left(\xi_{a} u_{2}\right) \wedge \ldots \wedge u_{n}+\ldots \\
& +u_{1} \wedge \ldots \wedge\left(\xi_{a} u_{n}\right)
\end{aligned}
$$

where $\xi_{a} u=a u_{\uparrow}+a u_{\downarrow}, u=u_{\uparrow}+u_{\downarrow}$, is the decomposition associated to $E=E_{\uparrow} \oplus E_{\downarrow}$, and both $E_{\uparrow}$ and $E_{\downarrow}$ are identified with $\mathbf{C}^{\ell}$. This action corresponds to the general linear group $G L(\ell)$ action

$$
u_{1} \wedge \ldots \wedge u_{n} \mapsto\left(g u_{1}\right) \wedge \ldots \wedge\left(g u_{n}\right)
$$

where the element $g \in G L(\ell)$ is represented as $g u=g u_{\uparrow}+g u_{\downarrow}$. The explicit isomorphism with $\mathbf{C}^{\ell}$ furnished by the bases (2.3) and (2.4) assigns to the matrix $a=\left(a_{i j}\right)$ the operator

$$
\xi_{a}=\sum_{i, j=1}^{\ell} a_{i j}\left(x_{i} \frac{\partial}{\partial x_{j}}+y_{i} \frac{\partial}{\partial y_{j}}\right),
$$


and, in particular, the kinetic-energy and number operators $n_{i}$ are represented by the matrices $t=\left(t_{i j}\right)$ and $E_{i i}$ (the elementary matrix with 1 at the $i i$ entry and zero elsewhere). The operator associated to the elementary matrix $E_{i j}$ will be denoted $\xi_{i j}$.

We can see now that the existence of the above-mentioned quantum numbers for the hamiltonian is a consequence of the fundamental property of $A$, its behavior with respect to the operation of direct sum of subspaces. The isomorphism

$$
\Lambda\left(E_{\uparrow} \oplus E_{\downarrow}\right) \cong \Lambda\left(E_{\uparrow}\right) \otimes \Lambda\left(E_{\downarrow}\right)
$$

induces a decomposition

$$
\Lambda(E)=\bigoplus_{M, N}\left(\Lambda^{M}\left(E_{\uparrow}\right) \otimes \Lambda^{N}\left(E_{\downarrow}\right)\right),
$$

where the module $\Lambda^{M}\left(E_{\uparrow}\right) \otimes \Lambda^{N}\left(E_{\downarrow}\right)$ under the action of $g l(\ell)$ contains $M$ spins up and $N$ spins down (equivalently, this subspace is characterized by the eigenvalues of $N$ and $S_{z}$ ). Under the action of the unitary group $U(\ell) \subset G L(\ell)$ (which preserves the canonical anticommutation relations, since it maps orthonormal bases of $E=E_{\uparrow} \oplus E_{\downarrow}$ into orthonormal bases) one obtains a decomposition of the moduli $\Lambda^{M}\left(E_{\uparrow}\right) \otimes \Lambda^{N}\left(E_{\downarrow}\right)$ into irreducible subspaces [3], the highest-weight moduli which are orbits of the dominant vectors $x_{1} x_{2} \ldots x_{M} y_{1} y_{2} \ldots y_{N}$ under the lower triangular algebra of $g l(\ell)$. Since the hamiltonian depends only on the $g l(\ell)$ action, these subspaces are also stable, and the Casimir operator

$$
\Delta=-\frac{1}{2} \sum_{i, j=1}^{\ell} \xi_{i j} \xi_{j i}
$$

is a multiple of the identity on each one. This operator can be written as a function of the total spin operator $S$ and the number of particles operator $N$ :

$$
\Delta=S^{2}+\frac{1}{4} N^{2}-\left(\frac{1}{2} \ell+1\right) N,
$$

so the irreducible subspaces are indexed by the total spin $s$. It is a standard result in representation theory [3] that these irreducible subspaces can be equally described as the kernels of the spin operator $S_{+}$for $M \geqslant N$, a fact observed in Ref. [5] for the solutions of the Bethe ansatz in the one-dimensional Hubbard model. We have thus obtained a quite explicit description of the reduction of $H$ in terms of the representations of the Lie algebra $g l(\ell)$.

\section{The Clifford algebra}

The space of states admits a second realization which leads to a different description of the Hubbard model. Since $E_{\downarrow} \cong E_{\downarrow}^{*}$ via the scalar product, one obtains the isomorphism

$$
\Lambda(E) \cong \Lambda\left(E_{\uparrow}\right) \otimes \Lambda\left(E_{\downarrow}\right) \cong \Lambda\left(E_{\uparrow}\right) \otimes \Lambda\left(E_{\downarrow}\right)^{*}
$$


so that

$$
\mathcal{H} \cong C(\ell)
$$

the Clifford algebra of the space $\mathbf{C}^{\ell}$, i.e., the space of linear transformations of $\Lambda\left(\mathbf{C}^{\ell}\right)$ [3]. Our next goal is to obtain an explicit isomorphism such that the action of the hamiltonian be as simple as possible. Since $\mathcal{H}=\Lambda\left(\mathbf{C}^{\ell}\right) \otimes A\left(\mathbf{C}^{\ell}\right)$, the states are linear combinations of vectors of the form $u \otimes v, u, v \in \Lambda\left(\mathbf{C}^{\ell}\right)$. Let us fix an orientation in $\Lambda\left(\mathbf{C}^{\ell}\right)$, i.e., pick a volume element $\Omega \in A^{\ell}\left(\mathbf{C}^{\ell}\right)$, and let us associate to $v$ the operator $v \rightarrow \Omega^{+} v$, so that $u \otimes v \rightarrow u \otimes \Omega^{+} v$ or simply $u \Omega^{+} v$ if we consider $u$ and $v$ as multiplication operators in $\Lambda\left(\mathbf{C}^{\ell}\right)$. Given an orthonormal basis $\left\{x_{1}, \ldots, x_{\ell}\right\} \subset \mathbf{C}^{\ell}$ and $\Omega=x_{1} \ldots x_{\ell}$, to the standard basis $\left\{x^{\alpha} \otimes x^{\beta}\right\} \subset A\left(\mathbf{C}^{\ell}\right) \otimes \Lambda\left(\mathbf{C}^{\ell}\right)$ of states with $|\alpha|=\alpha_{1}+\ldots+\alpha_{\ell}$ spins up and $|\beta|=\beta_{1}+\ldots+\beta_{\ell}$ spins down there corresponds the basis of operators

$$
x^{\alpha} \Omega^{+} x^{\beta}: \Lambda^{\ell-|\beta|}\left(\mathbf{C}^{\ell}\right) \rightarrow \Lambda^{|\alpha|}\left(\mathbf{C}^{\ell}\right)
$$

where

$$
\Omega^{+}=\frac{\partial}{\partial x_{\ell}} \cdots \frac{\partial}{\partial x_{1}}
$$

The scalar product in $C(\ell)$ is fixed by declaring the basis $x^{\alpha} \Omega^{+} x^{\beta}$ orthonormal, so that $(X, Y)=\operatorname{tr}\left(X^{+} Y\right)$. The idea of eliminating one of the species of spins has been previously applied by Lieb [6], who used the matrix form of the hamiltonian in the basis $x^{\alpha} \otimes x^{\beta}$ to prove two theorems on the spin of the ground state of the Hubbard model. We add to this use of spinless fermions the new representation just defined, which is the key ingredient to write in a simple way complex many-particle states.

To calculate the form of the hamiltonian in the Hilbert space $C(\ell)$ it is enough to study the behavior of the representation of $g l(\ell)$ under the isomorphism just defined since, as we said in Section 2, the Hubbard hamiltonian belongs to the enveloping algebra of $g l(\ell)$. Such form is determined by the commutativity of the following diagram:

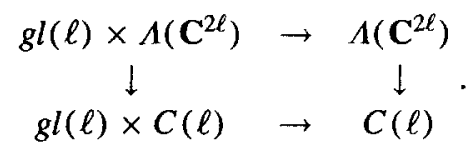

Each matrix $a$ acts on $\Lambda\left(\mathbf{C}^{2 \ell}\right) \cong \Lambda\left(\mathbf{C}^{\ell}\right) \otimes \Lambda\left(\mathbf{C}^{\ell}\right)$ according to $\xi_{a}(u \otimes v)=\left(\xi_{a} u\right) \otimes v+$ $u \otimes\left(\xi_{a} v\right)$, to which corresponds the following operator of $C(\ell)$ :

$$
\left(\xi_{a} u\right) \Omega^{+} v+u \Omega^{+}\left(\xi_{a} v\right) .
$$

Considering the natural inclusion $g l(\ell) \subset C(\ell)$, this operator in turn can be rewritten

$$
\left[\xi_{a}, u \Omega^{+} v\right]+(\operatorname{tr} a) u \Omega^{+} v
$$

which differs from the natural action of $g l(\ell)$ by a multiple of the identity associated to the trace of $a$. As a consequence, the action of the Hubbard hamiltonian on an element $X \in C(\ell)$ is 


$$
H(X)=[K, X]+\frac{1}{2} \sum_{i=1}^{\ell} V_{i}\left[n_{i},\left[n_{i}, X\right]\right]-\frac{1}{4}\left(\sum_{i=1}^{\ell} V_{i}\right) X,
$$

where $K$ represents the matrix $t_{i j}$, i.e.,

$$
K=\sum_{i, j=1}^{\ell} t_{i j} x_{i} \frac{\partial}{\partial x_{j}}
$$

and

$$
n_{i}=x_{i} \frac{\partial}{\partial x_{i}}
$$

denotes now the number operator for spinless fermions in $\Lambda\left(\mathbf{C}^{\ell}\right)$. Note that in this form, half the variables from the original problem have been eliminated.

Similarly, let us consider now the form of the generators of the spin algebra (1.4). The action of $N$ on a state $X$ is a straightforward application of Eq. (3.1). Since the corresponding matrix is the identity in $\mathbf{C}^{\ell}$,

$$
n=\xi_{\text {id }}=\sum_{i=1}^{\ell} x_{i} \frac{\partial}{\partial x_{i}}
$$

one obtains immediately

$$
N(X)=[n, X]+\ell X .
$$

The action of $S_{z}$ is also straightforward:

$$
S_{z}(X)=\frac{1}{2}(n X+X n-\ell X) \text {. }
$$

The form of the third generator, $S_{+}$, is more involved and requires a brief calculation. The action of $S_{+}$on a state $x^{\alpha} y^{\beta} \in \Lambda\left(\mathbf{C}^{2 \ell}\right)$ is

$$
S_{+}\left(x^{\alpha} y^{\beta}\right)=\sum_{i=1}^{\ell} x_{i} \frac{\partial}{\partial y_{i}} x^{\alpha} y^{\beta}=\sum_{i=1}^{\ell} x^{\alpha} x_{i} \frac{\partial}{\partial y_{i}} y^{\beta},
$$

while to this basis vector there corresponds the state $x^{\alpha} \otimes x^{\beta} \in \Lambda\left(\mathbf{C}^{\ell}\right) \otimes \Lambda\left(\mathbf{C}^{\ell}\right)$, on which

$$
S_{+}\left(x^{\alpha} \otimes x^{\beta}\right)=\sum_{i=1}^{\ell} x^{\alpha} x_{i} \otimes \frac{\partial x^{\beta}}{\partial x_{i}} .
$$

Then, as follows from Eq. (2.1) and the fact that $\Omega^{+} \partial / \partial x_{i}=0$, in the Clifford algebra we have,

$$
S_{+}\left(x^{\alpha} \Omega^{+} x^{\beta}\right)=\sum_{i=1}^{\ell} x^{\alpha} x_{i} \Omega^{+} \frac{\partial x^{\beta}}{\partial x_{i}}=\sum_{i=1}^{\ell} x^{\alpha} x_{i} \Omega^{+} x^{\beta} \frac{\partial}{\partial x_{i}}(-1)^{|\beta|+1} .
$$

Hence, 


$$
S_{+}\left(x^{\alpha} \Omega^{+} x^{\beta}\right)=(-1)^{|\alpha|+|\beta|+1} \sum_{i=1}^{\ell} x_{i} x^{\alpha} \Omega^{+} x^{\beta} \frac{\partial}{\partial x_{i}}
$$

and the actions of $S_{+}$and $S_{-}$on a state $X$ with $N$ particles are

$$
\begin{aligned}
& S_{+}(X)=(-1)^{N+1} \sum_{i=1}^{\ell} x_{i} X \frac{\partial}{\partial x_{i}}, \\
& S_{-}(X)=(-1)^{N+1} \sum_{i=1}^{\ell} \frac{\partial}{\partial x_{i}} X x_{i} .
\end{aligned}
$$

Greater flexibility in the description of the states is achieved with the introduction of a new basis of the Clifford algebra $C(\ell)$ given by the $2^{\ell} \times 2^{\ell}=\operatorname{dim} C(\ell)$ linearly independent operators $x^{\alpha} \partial^{\beta} / \partial x^{\beta}$. The old basis $x^{\alpha} \Omega^{+} x^{\beta}$ can be written in terms of the new basis by using the canonical anticommutation relations (1.1)-(1.3) to transfer the $\partial / \partial x_{i}$ to the right of the $x_{i}$. Conversely, given $x^{\alpha} \partial^{\beta} / \partial x^{\beta}$ one can use the identity

$$
1=\prod_{i=1}^{\ell}\left(x_{i} \frac{\partial}{\partial x_{i}}+\frac{\partial}{\partial x_{i}} x_{i}\right)
$$

to write

$$
x^{\alpha} \frac{\partial}{\partial x^{\beta}}=x^{\alpha} \prod_{i=1}^{\ell}\left(x_{i} \frac{\partial}{\partial x_{i}}+\frac{\partial}{\partial x_{i}} x_{i}\right) \frac{\partial}{\partial x^{\beta}}
$$

as a linear combination of the $x^{\alpha} \Omega^{+} x^{\beta}$. The main advantage of the new basis is the existence of an adapted decomposition of the Clifford algebra $C(\ell)$ as a direct sum of subspaces invariant under the action of the hamiltonian:

$$
\begin{aligned}
\Gamma_{A B}(\ell) & =\mathbf{C}\left\{x^{\alpha} \frac{\partial^{\beta}}{\partial x^{\beta}},|\alpha|=A,|\beta|=B\right\}, \\
C(\ell) & =\bigoplus_{A, B=0}^{\ell} \Gamma_{A B}(\ell) .
\end{aligned}
$$

A state $X \in \Gamma_{A B}(\ell)$ has $A-B+\ell$ particles, since

$$
N\left(x^{\alpha} \frac{\partial^{\beta}}{\partial x^{\beta}}\right)=\left[n, x^{\alpha} \frac{\partial^{\beta}}{\partial x^{\beta}}\right]+\ell x^{\alpha} \frac{\partial^{\beta}}{\partial x^{\beta}}=(|\alpha|-|\beta|+\ell) x^{\alpha} \frac{\partial^{\beta}}{\partial x^{\beta}} .
$$

The operator $X \rightarrow\left[n_{i},\left[n_{i}, X\right]\right]$ gives the number of pairs plus the number of holes at the site $i$ (it is easy to check that $x^{\alpha} \Omega^{+} x^{\beta} \rightarrow\left(\alpha_{i}+\beta_{i}-1\right)^{2} x^{\alpha} \Omega^{+} x^{\beta}$ ), and the total number of bosons in the state $x^{\alpha} \partial^{\beta} / \partial x^{\beta}$ is given by

$$
\sum_{i=1}^{\ell}\left[n_{i},\left[n_{i}, x^{\alpha} \frac{\partial^{\beta}}{\partial x^{\beta}}\right]\right]=\|\alpha-\beta\|^{2} x^{\alpha} \frac{\partial^{\beta}}{\partial x^{\beta}}
$$


with eigenvalue $\|\alpha-\beta\|^{2}=\|\alpha\|^{2}+\|\beta\|^{2}-2(\alpha, \beta)=A+B-2(\alpha, \beta)$. Consequently $A+B$ is the maximum number of bosons in $\Gamma_{A B}(\ell)$. The states in $\Gamma_{A B}(\ell)$ are not (in general) eigenvectors of $S_{z}$.

There is a convenient interpretation of the potential energy operator in terms of the weights of the vectors in $\Gamma_{A B}(\ell)$ under the action of the unitary group $U(\ell)$ on $C(\ell)$. The image of $x^{\alpha}$,

$$
g\left(x^{\alpha}\right)=g\left(x_{1}^{\alpha_{1}}\right) \ldots g\left(x_{\ell}^{\alpha_{\ell}}\right)
$$

for the action on $\Lambda\left(\mathbf{C}^{\ell}\right)$ allows us to write

$$
g\left(x^{\alpha} \frac{\partial^{\beta}}{\partial x^{\beta}}\right)=g\left(x^{\alpha}\right) \frac{\partial^{\beta}}{\partial g\left(x^{\beta}\right)} .
$$

In particular, the diagonal matrices $x_{i} \rightarrow \lambda_{i} x_{i},\left|\lambda_{i}\right|=1$ transform the state $x^{\alpha} \Omega^{+} x^{\beta} \rightarrow$ $\left(\lambda^{\mu} / \lambda^{\nu}\right) x^{\alpha} \Omega^{+} x^{\beta}$, where $|\mu|$ is the number of pairs and $|\nu|$ the number of holes. I.e., the weights of the $U(\ell)$ representation give the numbers of pairs and holes in a state. A generic operator $X$ transforms under the action of the diagonal subgroup according to $X \rightarrow \sum\left(\lambda^{\mu} / \lambda^{\nu}\right) X_{\mu \nu}$, where $X_{\mu \nu}$ is a state with $|\mu|$ pairs, $|\nu|$ holes, and $\ell+|\mu|-|\nu|$ particles. For example, $x_{1} \partial / \partial x_{2}$ has $\ell$ particles, one pair at the site 1 and one hole at the site 2 . The subspace $\Gamma_{A B}(\ell)$ is thus a modulus for the action of the unitary group just defined and the decomposition as a direct sum of irreducible subspaces yields highest weight moduli on which the hamiltonian acts. The Casimir operator for this representation, $\Delta(X)=-\frac{1}{2} \sum_{i, j=1}^{\ell}\left[\xi_{i j},\left[\xi_{j i}, X\right]\right]$ is

$$
\Delta(X)=S^{2}(X)+\frac{1}{4}\left(n^{2} X+X n^{2}\right)-\frac{1}{2} n X n-\frac{1}{2} \ell\left(\frac{1}{2} \ell+1\right) X,
$$

from where we readily get the formula

$$
\Delta=S^{2}+\frac{1}{4}(N-\ell)^{2}-\frac{1}{2} \ell\left(\frac{1}{2} \ell+1\right) .
$$

The operator

$$
S_{+}: \Gamma_{A B}(\ell) \rightarrow \Gamma_{A+1, B+1}(\ell),
$$

and the maximum weight vectors in $C(\ell)$ are

$$
x_{1} x_{2} \ldots x_{P} \frac{\partial}{\partial x_{Q-1}} \frac{\partial}{\partial x_{Q-2}} \ldots \frac{\partial}{\partial x_{\ell}},
$$

which for $P \geqslant Q$ are the dominant vectors in the old representation $x_{1} \ldots x_{P} y_{1} \ldots y_{Q}$ up to a sign.

A problem of obvious physical interest is the projection of the vectors in $C(\ell)$ into their spin components, which can be carried out with the decomposition $\Lambda\left(\mathbf{C}^{\ell}\right)=$ $\bigoplus_{k=0}^{\ell} A^{k}\left(\mathbf{C}^{\ell}\right)$.

The eigenvalues of the operator (3.2) (i.e., the number operator for spinless fermions with states in $A\left(\mathbf{C}^{\ell}\right)$, not to be confused with the total number of particles in the system) are $0,1,2, \ldots, \ell$ and its minimum polynomial is 


$$
n(n-1)(n-2) \ldots(n-\ell)=0 .
$$

Consequently we can define orthogonal projections $P_{k}: \Lambda\left(\mathbf{C}^{\ell}\right) \rightarrow \Lambda^{k}\left(\mathbf{C}^{\ell}\right)$ as

$$
P_{k}(n)=p_{k} \prod_{\substack{r=0 \\ r \neq k}}^{\ell}(n-r)
$$

where

$$
\frac{1}{p_{k}}=\prod_{\substack{r=0 \\ r \neq k}}^{\ell}(k-r)
$$

and any state $X \in C(\ell)$ is an operator $X: A\left(\mathbf{C}^{\ell}\right) \rightarrow A\left(\mathbf{C}^{\ell}\right)$ whose components $X_{a b}$ : $\Lambda^{b}\left(\mathbf{C}^{\ell}\right) \rightarrow \Lambda^{a}\left(\mathbf{C}^{\ell}\right)$ with respect to the previous decomposition, given by

$$
X_{a b}=P_{a}(n) X P_{b}(n),
$$

satisfy

$$
\begin{gathered}
N\left(X_{a b}\right)=(a-b+\ell) X_{a b}, \\
S_{z}\left(X_{a b}\right)=\frac{1}{2}(a+b-\ell) X_{a b} .
\end{gathered}
$$

That is to say, $X_{a b}$ is a state with $(a-b+\ell)$ particles and $\frac{1}{2}(a+b-\ell)$ third spin component.

\section{Elementary states}

In this section we draw a few direct consequences of the Clifford algebra representation. In particular we will see how this representation suggests several families of nontrivial half-filled states. Hereafter we set all the coefficients $V_{i}$ equal to $U$ and omit the constant term, so that the Schrödinger equation for the Hubbard hamiltonian becomes

$$
[K, X]+\frac{1}{2} U \sum_{i=1}^{\ell}\left[n_{i},\left[n_{i}, X\right]\right]=E X
$$

A first simple observation is that any function of $n$ is trivially a half-filled state with zero energy, whose third spin component can be seen with the $(\ell+1)$ projections $P_{a}(n)$ defined above. Since $S_{z}\left(P_{a}\right)=\frac{1}{2}(2 a-\ell) P_{a}$, the projection $P_{a}(n)$ has spin $\left(a-\frac{1}{2} \ell\right)$, $a=0,1, \ldots, \ell$. Furthermore,

$$
S_{+}\left(P_{a}\right)=(-1)^{\ell+1} \frac{p_{a}}{p_{a+1}}(a-\ell) P_{a+1}
$$

and $P_{0}(n)$ is the half-filled state with all the spins down. 
Another family of states that can be easily described in the new representation are the operators in $g l(\ell)$,

$$
\xi_{a}=\sum_{i, j=1}^{\ell} a_{i j} x_{i} \frac{\partial}{\partial x_{j}}
$$

For $r \neq s$ the state $x_{r} \partial / \partial x_{s}$ has $\ell$ particles with one pair in the site $r$ and one hole in the site $s$ (if $r=s$ it has neither pairs nor holes). These operators $\xi_{a}$ do not exhaust the family of half-filled states, which in fact is described by all the superpositions of $x^{\alpha} \partial^{\beta} / \partial x^{\beta}$ operators with $|\alpha|=|\beta|$. From a mathematical point of view, the half-filled states are nothing but the diagonal transformations with respect to the decomposition $\Lambda\left(\mathbf{C}^{\ell}\right)=\bigoplus_{k=0}^{\ell} \Lambda^{k}\left(\mathbf{C}^{\ell}\right)$. Among these there is a distinguished element, namely (3.2), that defines the kinetic energy and is the representative of the matrix $t$ (i.e., $K=\xi_{t}$ ). It has zero kinetic energy, and $U$ potential energy (i.e., $V(K)=U K$ ) since it is a superposition of states with one pair and one hole. The state $K$ is therefore a half-filled eigenstate of the hamiltonian with total energy $U$,

$$
H(K)=U K
$$

This result is valid independently of the structure of the hopping matrix $t$, the dimension of the lattice, or its bipartite character, i.e., the $\eta$ symmetry introduced by Yang $[7,8]$. The state $K$ is a particular instance of a more general family of eigenstates with the same energy $U$ : since for any matrix $a=\left(a_{i j}\right)$ one has the commutation rule

$$
\left[\xi_{a}, K\right]=\xi_{[a, t]},
$$

one can see immediately that

$$
H\left(\xi_{a}\right)=U \xi_{a}
$$

whenever $a$ commutes with $t$ and has zeros in the diagonal.

To calculate the spin components of the states $\xi_{a}$ we first note that for $r \neq s$, the state $\xi_{r s}=x_{r} \partial / \partial x_{s}$ belongs to the irreducible representation $\operatorname{sl}(\ell)$ which is the orbit associated to the highest weight vector $x_{1} \partial / \partial x_{\ell}$ under the action of the lower triangular algebra $\mathbf{C}\left\{x_{i+j} \partial / \partial x_{i}\right\}$ with spin $\frac{1}{2}(\ell-2)$. Since the projections $P_{a}(n)$ commute with the elements of $g l(\ell)$ we obtain that, unless $a=b, P_{a} \xi_{r s} P_{b}=P_{a} P_{b} \xi_{r s}=0$ and $S_{z}\left(\xi_{r s}^{a}\right)=$ $\frac{1}{2}(2 a-\ell) \xi_{r s}^{a}$, here $\xi_{r s}^{a}=P_{a} \xi_{r s} P_{a}=P_{a} \xi_{r s}$. That is to say, $\xi_{r s}^{a}$ is the projection onto the subspace with $S_{z}$ equal to $\frac{1}{2}(2 a-\ell)$. The eigenvalues of $S_{z}$ range from 1 to $\ell-1$, since $\xi_{r s}$ has one pair and one hole,

$$
\xi_{r s}=\sum_{a=1}^{\ell-1} \xi_{r s}^{a}
$$

(note that $\xi_{r s}$ vanishes on $\Lambda^{0}\left(\mathbf{C}^{\ell}\right) \cong \Lambda^{\ell}\left(\mathbf{C}^{\ell}\right) \cong \mathbf{C}$ ). Let us calculate now the relation between the different spin components $\xi_{r s}^{a}$ : we shall prove that 


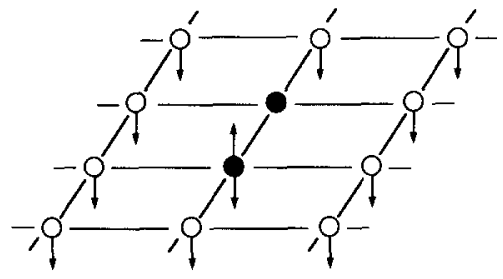

1 (a)

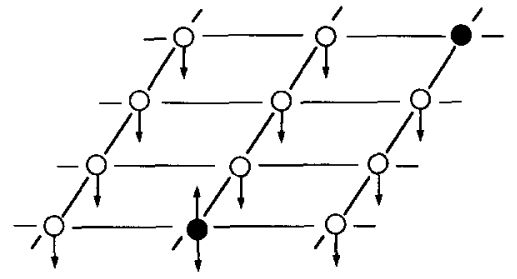

1 (b)

Fig. 1. (a) Typical state that enters in the linear combination of the eigenstate $K$. (b) State with one pair and one hole separated by a fixed number of links.

$$
S_{+}\left(\xi_{r s}^{a}\right)=\frac{p_{a}}{p_{a+1}} \frac{a(a-\ell)}{a+1}(-1)^{\ell} \xi_{r s}^{a+1} .
$$

Since $x_{j}(n-k)=[n-(k+1)] x_{j}$ we see that

$$
\begin{aligned}
S_{+}\left(\xi_{r s}^{a}\right) & =(-1)^{\ell+1} p_{a} \prod_{k=1, k \neq a-1}^{\ell+1}(n-k) \sum_{i=1}^{\ell} x_{i} \xi_{r s} \frac{\partial}{\partial x_{i}} \\
& =(-1)^{\ell+1} p_{a} \prod_{k=1, k \neq a-1}^{\ell+1}(n-k) \xi_{r s},
\end{aligned}
$$

is an operator with the structure $p_{a} Q_{a}(n) \xi_{r s}$. Decomposing $\xi_{r s}$ once more, we find

$$
S_{+}\left(\xi_{r s}^{a}\right)=(-1)^{\ell+1} p_{a} Q_{a}(n) \sum_{b=1}^{\ell-1} P_{b}(n) \xi_{r s}=(-1)^{\ell+1} p_{a} \sum_{b=1}^{\ell-1} Q_{a}(b) P_{b}(n) \xi_{r s},
$$

since $n P_{b}(n)=b P_{b}(n)$. The only nonvanishing value of $Q_{a}(b)$ is $Q_{a}(a+1)=a(a-$ $\ell) /\left[p_{a+1}(a+1)\right]$, hence our statement.

The state $\xi_{r s}$ is a superposition of eigenstates $\xi_{r s}^{a}$ of $S_{z}$ which can be obtained recursively with the operator $S_{+}$, so it suffices to analyze the lowest component of spin $\xi_{r s}^{1}$ in which there is a pair in the site $r$, a hole in the site $s$, and the remaining sites are occupied by spins down. The operator $\xi_{r s}^{1}$ is the restriction $\xi_{r s} \mid \Lambda^{1}\left(C^{\ell}\right)$ that maps $x_{r} \rightarrow x_{s}$, i.e., it is the elementary matrix

$$
(-1)^{s+\ell} x_{r} \Omega^{+} x_{1} x_{2} \ldots x_{s-1} x_{s+1} \ldots x_{\ell} .
$$

The state $K=\sum_{i, j=1}^{\ell} t_{i j} \xi_{i j}=\sum_{j=1}^{\ell} t_{j}(x) \partial / \partial x_{j}$ where $t_{j}(x)=\sum_{i=1}^{\ell} t_{i j} x_{i}$ is a superposition of the states $\xi_{i j}$. Its lowest spin component $K^{1}$ can be written as

$$
K^{1}=\sum_{j=1}^{\ell}(-1)^{j+\ell} t_{j}(x) \Omega^{+} x_{1} x_{2} \ldots x_{j-1} x_{j+1} \ldots x_{\ell}
$$

that in $A\left(\mathbf{C}^{2 \ell}\right)$ is represented by the state

$$
\psi_{K}=\sum_{j=1}^{\ell} t_{j}(x) \frac{\partial}{\partial y_{j}}\left(y_{1} y_{2} \ldots y_{\ell}\right)
$$


As an example exhibiting the essential aspects of $\psi_{K}$ in any dimension, consider a two-dimensional lattice. In that case $\psi_{K}$ is a superposition of states of the type shown in Fig. 1a.

If instead of $t$ we consider a matrix $q$ such that $[q, t]=0, q_{i i}=0$, performing a similar analysis we obtain the state

$$
\psi_{q}=q_{1}(x) y_{2} \ldots y_{\ell}+y_{1} q_{2}(x) \ldots y_{\ell}+\ldots+y_{1} y_{2} \ldots q_{\ell}(x)
$$

which comes from the dual vacuum $y_{1} y_{2} \ldots y_{\ell}$ through the flow defined by the vector field

$$
\sum_{j=1}^{\ell} q_{j}(x) \frac{\partial}{\partial y_{j}}
$$

In certain cases one can give an explicit description of the matrices in the centralizer of $t$ with vanishing diagonal entries. The simplest examples arise in connection with periodic lattices that for our purposes can be conveniently described in terms of the tensor product

$$
\mathbf{C}^{\ell}=\mathbf{C}^{\ell_{1}} \otimes \mathbf{C}^{\ell_{2}} \otimes \ldots \otimes \mathbf{C}^{\ell_{d}}
$$

for the case of a $d$-dimensional lattice. The corresponding kinetic energy matrix can be written as a sum $t=t_{1}+\ldots+t_{d}$ in which

$$
t_{j}=1_{1} \otimes \ldots \otimes\left(\tau_{j}+\tau_{j}^{-1}\right) \otimes \ldots \otimes 1_{d}
$$

(assuming that all the translations are independent). The action of the unitary operator $\tau_{j}$ on the chosen basis is $\tau_{j} e_{r}=e_{r+1}$, so that if we denote the generators of the lattice translations by

$$
\bar{\tau}_{j}=1_{1} \otimes \ldots \otimes \tau_{j} \otimes \ldots \otimes 1_{\ell}
$$

then

$$
\bar{\tau}_{1}^{m_{1}} \bar{\tau}_{2}^{m_{2}} \ldots \bar{\tau}_{d}^{m_{d}}
$$

commutes with $t$ and (unless all the $m_{j}$ vanish) has zeros in the diagonal for $0 \leqslant m_{j} \leqslant$ $\ell_{j}-1$. By the previous construction, this family of matrices determine a corresponding family of eigenstates of the hamiltonian which are superpositions of states with one pair and one hole separated by a constant number of links $\left(m_{1}\right.$ in the first direction, $m_{2}$ in the second direction, etc.) A typical example with $m_{1}=1$ and $m_{2}=3$ is presented in Fig. 1b. Similar states with "low-hole doping" have been studied in the context of high-temperature superconductivity [9].

\section{References}

[1] D.C. Mattis, ed., The many-body problem (World Scientific, Singapore, 1993). 
[2] V.E. Korepin and F.H. EBler, eds., Exactly solvable models of strongly correlated electrons (World Scientific, Singapore, 1994).

[3] W. Fulton and J. Harris, Representation theory (Springer, Berlin, 1991).

[4] F.A. Berezin, The method of second quantization (Academic Press, New York, 1966).

[5] F.H. Eßler, V.E. Korepin and K. Schoutens, Nucl. Phys. B 384 (1992) 431.

[6] E.H. Lieb, Phys. Rev. Lett. 62 (1989) 1201.

[7] C.N. Yang, Phys. Rev. Lett. 63 (1989) 2144.

[8] C.N. Yang and S.C. Zhang, Mod. Phys. Lett. B 4 (1990) 759.

[9] E. Dagotto, Rev. Mod. Phys. 66 (1994) 763. 